Mirai. Estudios Japoneses

ISSN-e: 2531-145X

https://dx.doi.org/10.5209/mira.63067

\title{
Diplomacia de las muñecas japonesas en los siglos XIX y XX: Prensa, intercambios y exposiciones.
}

\author{
María Gutiérrez Montañés ${ }^{1}$
}

Recibido: 25 de enero de 2019/ 15 de abril de 2019

Resumen. Muchos han sido los momentos de encuentro entre Japón y España, así como los objetos testigo de estas relaciones que este 2018 cumplen 150 años. Entre tales objetos, se encuentran las muñecas tradicionales niponas que fueron exportadas a España ya desde los períodos Meiji (1868-1912) y Taishō (1912-1926) y que hoy forman parte de los fondos de distintas colecciones públicas y privadas de nuestro país.

Dejando aparte su coleccionismo, es objetivo de este articulo esbozar la historia de la presencia de estas de piezas en nuestro país a través de las noticias que nos brindan las revistas ilustradas y la prensa española a lo largo estos 150 años de relaciones, tratando aspectos como intercambios, notas de prensa acerca de la historia y procedencia de estas piezas y textos que aluden a exposiciones; ya que las fuentes mencionadas nos informan de que las muñecas niponas han sido recurrentes en muestras desde mediados del siglo XIX hasta la actualidad, así como objeto de interés y estudio por parte de artículos y reportajes.

Por otro lado, y en relación con esta presencia, incluimos también algunos intercambios, la mayoría de ellos pertenecientes a una vertiente diplomática, aunque citaremos otros ejemplos anecdóticos e igualmente interesantes. Como piedra angular, definimos el caso de las Friendship Dolls y el intercambio que tuvo lugar entre Japón y Estados Unidos para hablar de casos mucho más cercanos que van desde la boda del príncipe Chichibu hasta el hermanamiento de Pamplona con Yamaguchi, todo ello con estas piezas presentes.

Palabras clave: Muñeca; ningyō; Japón; diplomacia; prensa.

\section{[en] Diplomacy of Japanese dolls in the 19th and 20th centuries: Press, exchanges and exhibitions.}

Abstract. The moments of encounter between Japan and Spain have been many, as well as the objects
which witnessed these exchanges. Exchanges that in 2018 celebrated their 150 anniversary. Among
these objects we can find traditional Japanese dolls that were exported to Spain from the Meiji (1868-
1912) and Taishō (1912-1926) eras, and that now, form part of the deposits of different public and
private collections in Spain. The objective of this article is to trace the history of the presence of this
type of pieces in our country through the news that the illustrated magazines and the Spanish press give
us throughout these 150 years of relationships referring to some diplomatic exchanges, the history and
origins of these dolls, and some articles about exhibitions since the sources mentioned above inform
us that traditional Japanese dolls have been the subject of a lot of exhibitions from the mid-nineteenth
century to the present day and have been objects of study in articles and researches.
On the other hand, we also include some exchanges, most of them diplomatic, although we will include
other equally interesting examples. As a cornerstone, we define the Friendship Dolls case and the ex-

1 Universidad de Zaragoza.

E-mail: mariagutierrezmontanes@gmail.com 
change between Japan and United States to, later, write about other examples that took place in Spain, like Prince Chichibu's wedding or the establishment of a sister city agreement between Pamplona and Yamaguchi. These are some examples where Japanese Dolls have been present.

Keywords: Doll; ningyō; Japan; diplomacy; press.

Sumario. 1. Introducción: Definición de ningyō y evolución posterior. 2. Recepción y presencia de ningyō. 3. Conclusiones. Bibliografía.

Cómo citar: Gutiérrez Montañes, M. (2019). Diplomacia de las muñecas japonesas en los siglos XIX y XX: Prensa, intercambios y exposiciones, en Mirai. Estudios Japoneses 3(2019), 167-178.

\section{Introducción: Definición de ningȳō y evolución posterior.}

El término que se utiliza en Japón para denominar a las muñecas tradicionales es ningyo (人形), formado por los kanji, nin (人, persona, humano) y gyo (形, forma), por lo que la palabra vendría a significar "forma humana" "forma de persona". ${ }^{2}$ Este término fue traducido al inglés como doll, vocablo que ha llevado a que en Occidente, las ningyō se identificaran con nuestra definición de muñeca como juguete infantil. Sin embargo, estos objetos no sólo responden a esta acepción, ya que, además de ser en algunos casos un juguete, desempeñan diversas funciones y ofrecen distintos significados, siendo con frecuencia amuletos protectores contra los malos espíritus, objetos propiciatorios de buen augurio, modelos de conducta con su pertinente función didáctica, objetos de apreciación estética o incluso meros souvenirs. ${ }^{3}$

La mayor parte de las muñecas se realizan con materiales como la madera, cerámica, paja o papel maché que, en la mayoría de los casos, reciben un acabado mediante una pasta denominada gofun, compuesta por conchas marinas trituradas y un aglutinante procedente de la espina de pez denominado nikawa, que aporta un acabado blanco, liso y pulido. ${ }^{4}$ Este acabado, además, queda completado con la inclusión de policromía, cabello natural y ojos de cristal y ricos atuendos realizados en tejidos como seda y algodón.

Con independencia del remoto origen de estas figuras antropomorfas, el mayor desarrollo de su producción tuvo lugar en el período Edo (1603/1615-1868), una época en la que Japón vivió en paz y prosperidad bajo el gobierno de los shōgun de la familia Tokugawa, que lograron unificar el país bajo su autoridad. Esta etapa se caracterizó por el férreo control al que se sometió a todos los sectores de la sociedad (especialmente a los señores feudales o daimyō), que garantizó la estabilidad interior, el aislamiento del país y el desarrollo del arte y las artesanías, así como del comercio, que trajo consigo la prosperidad económica y el auge y expansión de la burguesía. ${ }^{5}$

Estas circunstancias históricas, además, propiciaron el desarrollo de la producción y el consumo de ningyō: Por una parte, en las distintas regiones del archipiélago

\footnotetext{
Yamada, Tokubei (1955): p. 1.

Gómez Pradas, Muriel (2011): vol.1, p. 108.

Pate, Alan Scott (2005): p. 14.

Whitney, John (ed.) (1991): vol. 4, pp. 1-6.
} 
hubo un auge de talleres artesanales y excelentes autores dentro de la creación de estas figuras; por otra, el clima de paz y estabilidad propició su consumo, que se extendió entre distintas capas de la sociedad. En esta época se produjo una mayor variedad tipológica, incluyendo ejemplares realizados en materiales más baratos que permitían su compra a más sectores de la sociedad. En consecuencia, la mayor demanda hizo que avanzara la calidad y el virtuosismo de su ejecución. ${ }^{6}$

Esta producción se mantuvo en la era Meiji (1868-1912) y también en el período Taishō (1912-1926). En estas etapas, el descubrimiento y la fascinación que produjo Japón en los países occidentales, y que dio lugar al Japonismo (segunda mitad del siglo XIX y primeras décadas del XX), llevó consigo la importación de este tipo de artesanía, que fue dada a conocer tempranamente a través de las exposiciones universales e internacionales. Su éxito fue tal, que pronto estas muñecas fueron vendidas en tiendas especializadas, donde fueron adquiridas por coleccionistas. ${ }^{7}$ Es cierto que determinados tipos de piezas, como las estampas ukiyo-e, las cerámicas, o los objetos de laca y marfil, fueron especialmente valorados $;{ }^{8} \sin$ embargo, también las muñecas japonesas fueron compradas y tuvieron su lugar en las casas de aquella época, tanto en las vitrinas de los salones (las más caras y exquisitas) como en los cuartos de juego de las niñas. Igualmente, y estrechamente relacionado con el tema que nos ocupa, no podemos dejar de destacar el papel de estas piezas como protagonista de varios intercambios, algunos de carácter diplomático, además de exposiciones (tanto especializadas como otras muestras de carácter general centradas en el arte tradicional nipón), que quedan reflejadas en la prensa española del momento. Al igual que ocurre en los casos anteriores, se trata de ricos ejemplares pertenecientes a unas tipologías concretas que veremos con posterioridad.

\section{Primeros indicios de coleccionismo}

No es extraño que las ningyō tuvieran un éxito inusitado: por un lado, en Occidente se pusieron de moda las muñecas de biscuit (porcelana sin esmaltar) que representaban figuras infantiles (bebés o infantes de escasa edad), destinadas a las niñas de familias pudientes. Probablemente, por esta razón, la tipología que más llegó a Occidente fueron las Ichimatsu-ningyō, muñecas que representan a niños y niñas japoneses ataviados con el kimono tradicional que, vendría a cumplir una función como juguetes. A esto hemos de añadir la fascinación por lo japonés y la más que evidente calidad técnica de estas piezas, lo que llevó a que surgieran buen número de personas que las quisieran adquirir como objeto decorativo. A este respecto y teniendo en cuenta nuestra afirmación anterior, también debemos referirnos a las Ishō-ningyō o muñecas de vestir, que, como bien indica su nombre, destacan por lo cuidado de sus atuendos y peinados, siendo un fiel reflejo de la tradición nipona y una excelente figura para la decoración del hogar.

De esta forma, a pesar de que las ningyō son concebidas originalmente como un objeto de carácter ritual -como ya hemos comentado en su definición-, en Occidente pierden esta concepción para pasar a convertirse en piezas de colección, decoración

\footnotetext{
Yamada, Tokubei (1955): Japanese Dolls..., op. cit., p. 12.

Pate, Alan Scott: "Sosaku Ningyō: The Birth of The Japanese Art Doll”. En Antique Japanese Dolls. http:// www.antiquejapanesedolls.com/pub_artinfocus/sosaku_ningyo/sosaku_ningyo.html [Consulta: 23/01/2018].

8 Barlés Báguena, Elena (2012): pp. 95-156.
} 
o bien en un mero juguete. Esta valoración, que irá cambiando con el paso del tiempo y la aparición de estudios centrados en arte japonés, viene a dar explicación, por un lado a su papel como objeto diplomático y a su presencia en exposiciones. Si bien, debemos destacar, como atestiguan diversas fuentes, que estas actividades en nuestro país tienen una fecha posterior, llegando a dilatarse incluso hasta la década de los 70 y 80 del siglo $\mathrm{XX}$.

\section{Prensa y publicidad}

Respecto a su impacto en la prensa, aparecen un número significativo de artículos que mencionan estos objetos, muchos de ellos de gran utilidad para el estudio que estamos desarrollando. No obstante, en la mayoría de los casos, encontramos este tipo de referencias en revistas y publicaciones vinculadas con el coleccionismo y las antigüedades. Asimismo, aparecen en la prensa diaria y en las revistas ilustradas de la época. ${ }^{9}$ Sin embargo, la mayor parte de la información que se va a incluir en prensa está estrechamente vinculada con las ilustraciones publicitarias, de modo que se aporta una visión naïf y centrada en el mundo de la infancia; aunque no podemos dejar de destacar otros textos que nos hablan de una consideración que va más allá, refiriéndose, por ejemplo, a la admiración que despierta la industria juguetera nipona. ${ }^{10}$ Por otro lado, y en relación con lo ya comentado, estas piezas aparecen a menudo en el mundo de las artes gráficas (ilustraciones e impresos), donde encontramos representaciones de niñas occidentales acompañadas de sus muñecas en muy diversas situaciones, todas ellas vinculadas con la cotidianidad y el ámbito doméstico. ${ }^{11}$ También en la publicidad se distingue el auge de motivos orientales, y más concretamente la imagen de ningyō, que se va a extender hasta la década de los años 20 del siglo XX. En estos casos, nos encontramos una temática similar a las ilustraciones anteriores añadiéndose el logo del producto o un breve eslogan para convertirlas en un anuncio. Las muñecas japonesas se relacionan con la promoción de productos alimenticios de todo tipo, u otros de belleza e higiene dirigidos al público femenino. ${ }^{12}$

\section{Recepción y presencia de ningyo}

A la hora de estructurar nuestro estudio, vamos a centrarnos en dos momentos concretos que van a tener gran importancia: por un lado nos referiremos a la época del Japonismo (segunda mitad del siglo XIX y primeras décadas del XX) para después tratar su evolución posterior llegando prácticamente hasta la actualidad.

El interés que despertó Japón en Occidente vino de la mano de su apertura. A este respecto, debemos mencionar la figura del comodoro Matthew Perry que a su vuelta trajo consigo dos ejemplares de muñecas japonesas pertenecientes a la tipología Ichimatsu-ningyō que hemos definido con anterioridad. A partir de este momento, las

\footnotetext{
9 Para más información véase: Almazán Tomás, David (1999).

10 Anónimo (1970): “Castellón es en estos días un polícromo bazar de juguetes”, Mediterráneo: Prensa y Radio del Movimiento, Castellón, 04/01/1970.

11 A título de ejemplo véase: Shoaf, Judy (2003): The Japanese doll on the Western Toyshelf [página WEB], http:// users.clas.ufl.edu/jshoaf/Jdolls/jdollwestern/2005/index.html [Consulta: 23/01/2019].

12 A título de ejemplo véase: Shoaf, Judy (2003): The Japanese doll on the Western Toyshelf [página WEB], http:// users.clas.ufl.edu/jshoaf/Jdolls/jdollwestern/Ads/addoll.htm [Consulta: 23/01/2019].
} 
potencias occidentales van a apresurarse a establecer contactos de muy diversa índole (diplomáticos, comerciales, etc.) con el archipiélago nipón, lo que trajo consigo la importación de diversas artesanías que pasaron a venderse en tiendas especializadas.

El Japonismo histórico en el caso español, ámbito en el que fundamentalmente centramos nuestra investigación, se desarrolló desde mediados del siglo XIX hasta la Guerra Civil española. ${ }^{13}$ Los contactos directos entre España y Japón en esta época fueron limitados, ya que nos encontramos en un momento de declive para nuestro país. De esta manera, a la hora de hablar de los primeros tratados de comercio y amistad que se establecieron entre ambos países, hemos de retrotraernos al año 1868, momento en el que ambos países firman en Kanagawa un tratado de amistad, comercio y navegación que permitió establecer un cuerpo diplomático, ${ }^{14}$ primero en Yokohama y luego en Tokio. Sin embargo, la actividad comercial fue bastante escasa debido a la falta de infraestructuras. A pesar de ello, todo va a empezar a cambiar durante la década de los 80 , momento en el que establecer contactos con Japón va a convertirse en una importante preocupación, por lo que se va a intentar llevar a cabo una ruta directa, sin intermediarios. Gracias a este comercio directo, y al que se produjo indirectamente -que fundamentalmente venía de Francia-, llegaron a España numerosos productos y objetos artísticos, entre ellos, muñecas tradicionales, que fueron adquiridas por artistas, literatos, eruditos, diplomáticos, empresarios y comerciantes, además de burgueses contagiados de la fascinación por lo japonés. ${ }^{15}$

En relación con esto, debemos subrayar el papel de las exposiciones universales, sobre todo las que tuvieron lugar en Barcelona en los años 1888 y 1929, que podemos conocer gracias a una serie de fotografías y artículos referentes a las mismas. ${ }^{16}$ En ambos casos, el archipiélago nipón participó mostrando sus productos, entre los que encontramos ningyō.

Tras estos primeros contactos comerciales y exposición, la aparición de estas muñecas en prensa fue algo común, siendo uno de los pilares más importantes para la difusión de estas piezas y la industria juguetera japonesa. En los primeros textos, podemos encontrar toda una serie de temas recurrentes que se repiten con posterioridad: Por un lado, debemos hablar de creaciones de carácter literario, ya sean versos o cuentos en prosa, que muchas veces se acompañan de ilustraciones o fotografías. Como ejemplos de ello tenemos el pequeño cuento que aparece en El Camarada (1888) ${ }_{17}^{17}$ acerca de unas niñas que se asombran ante la rareza de un ejemplar japonés, este relato se acompaña de una ilustración donde aparece representada una Ichimatsu-ningy $\overline{0}$.

Un segundo tipo de artículos son aquellos que abordan un análisis de lo que se denomina como "la historia de las muñecas", donde los ejemplares japoneses son resaltados tanto por su antigüedad como por su calidad y preciosismo. Estos ensayos también se ilustran mediante fotografías o dibujos, identificando las tipologías de las muñecas con mayor o menor acierto. A este respecto, citaremos el titulado "La vida contemporánea" (1906) ${ }_{18}^{18}$ en La Ilustración Artística, donde se pone de relieve el cuidado y exquisitez de las muñecas tradicionales japonesas que son entendidas

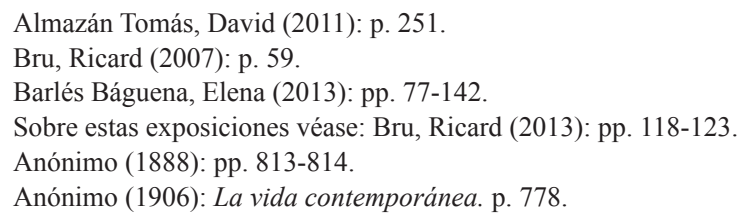


como un objeto artístico. Gracias a este tipo de textos más completos y reflexivos, la frontera entre el juguete y el objeto con otras funciones (decorativas, simbólica, didácticas, etc.) es mucho menos clara en el caso de estos ejemplares nipones. También, y como un primer caso de intercambio protagonizado por estas muñecas, hemos de comentar brevemente la unión del príncipe Chichibu, o Yasuhito, con Matsudaira Setsuko de la que se hace eco la prensa española, definiendo la llamada "ofrenda de la muñeca" durante la que el príncipe "regalarle varias muñecas iguales a las que todo el mundo en el Japón adquiere para la fiesta de la muñeca, una de las festividades más alegres del país." ${ }^{19}$ En este caso, se trata de una alusión clara al Hinamatsuri o Día de las niñas, ${ }^{20}$ en el que se lleva a cabo una pequeña muestra de muñecas que vendría a representar a la corte imperial; esta es una festividad que también aparece comentada en la propia prensa de nuestro país en numerosas ocasiones. ${ }^{21}$

Si bien, también podemos citar menciones mucho más escuetas, que identifican a estas muñecas como parte de los objetos donados por las mujeres participantes en determinadas ferias y subastas de carácter benéfico. ${ }^{22}$

Por último, resulta curioso que en las revistas de la época, en especial en las ilustradas, se publicasen ilustraciones y pinturas japonistas, representando objetos como ningyō. Así lo vemos en la pintura Un público indulgente del artista alemán Theo Schumtz-Baudiss (1859-?), en La Ilustración Artística (1894). ${ }^{23} \mathrm{O}$ el de la ilustración ¿Porqué crecerá?, de Ramírez, publicada en la revista Nuevo Mundo (1916). ${ }^{24}$ Por otro lado, tenemos el caso de la obra Chinoserías, pintura realizada por el malagueño José Blanco Coris (1862-1946), ${ }^{25}$ que se publicó en la revista Nuevo Mundo y en La Esfera. ${ }^{26}$ Un ejemplo significativo, entre otros, es el caso del anuncio del jabón Flores del Campo, publicado en la revista Nuevo Mundo (1914). ${ }^{27}$

Como ya hemos comentado al principio de este apartado, procedemos a tratar el segundo momento que nos resulta de interés a la hora de hablar de estas piezas como objeto diplomático y testigo de los contactos establecidos entre Japón y nuestro país. Nos referimos a la época posterior a la época al Japonismo y a la continua presencia de alusiones a estas piezas.

Para ello, en primer lugar, hemos de referirnos brevemente a un curioso episodio que nos transporta a Estados Unidos, y que hace alusión a estos intercambios diplomáticos. Ya hemos comentado que nuestro punto central es España, si bien, no podemos dejar de lado este primer encuentro por su importancia y por ser un caso pionero que pone de relieve el uso de estas ningyō como mensajeras de paz. ${ }^{28}$ Este fue un momento de gran presencia de inmigrantes japoneses, lo que trajo consigo un

19 Anónimo (1928): p. 1.

20 El Hinamatsuri o Día de las Niñas se celebra en Japón el tercer día del tercer mes. Se trata de una festividad popular en la que se exhibe un conjunto de muñecas a modo de representación de la corte imperial con una función didáctica y ceremonial. De este modo, las niñas aprenden una serie de comportamientos que se consideran positivos para su educación (a estar tranquilas, elegantes y bonitas, igual que las muñecas) así como se pone de relieve la importancia y el respeto hacia las tradiciones. Gómez Pradas, Muriel (2010): pp. 417-433.

21 Hardenberg (1904): p. 460; The Japan Chronicle (1916); El Progreso: Diario liberal (1916); Anónimo (1943).

22 Anónimo (1917).

23 Anónimo (1906): La Ilustración Artística, p. 643.

24 Anónimo (1916): Nuevo Mundo, 25 /08/1916.

25 Almazán Tomás, David (2004): pp. 503-522.

26 Anónimo (1929): Nuevo Mundo, 26/04/1929. Anónimo (1929): La Esfera, 27/04/1929.

27 "Flores del Campo", imagen de Joaquín Xauradó. Nuevo Mundo, Madrid, 05/09/1914.

28 Para más información acerca de las Friendship dolls véase: Pate, Alan Scott (2016). 
sentimiento de desconfianza y discriminación: muchos eran los americanos que criticaban a los japoneses por el cobro de salarios mucho más bajos, y, en consecuencia, porque arrebataran puestos de trabajo a los americanos. Por ello, hacia 1905, concretamente en San Francisco, se formó la Asiatic Exclusion League (Liga de la Exclusión Asiática), ${ }^{29}$ lo que supuso el inicio de un importante movimiento anti-japonés. Considerando esta situación, el presidente Theodore Roosevelt negoció lo que se conoce como el Gentlemen's Agreement (Acuerdo entre caballeros), ${ }^{30}$ de modo que el gobierno de Japonés se comprometió a detener la emisión de pasaportes a los trabajadores, frenando así la inmigración japonesa a Estados Unidos. Finalmente, en el año 1924 el presidente Calvin Coolidge firmó el Inmigration Act (Acto de Inmigración) ${ }^{31}$ que terminó definitivamente con las migraciones asiáticas (y concretamente japonesas) a Estados Unidos.

En este momento, entra en escena una figura fundamental que mostraba su desacuerdo con este rechazo y limitaciones hacia Japón, el Dr. Sidney Gulick (18601945), creador de un programa que permitiera comunicarse a los ciudadanos de ambas naciones, sobre todo los más jóvenes. Nos referimos al Committee on World Friendship Among Children (Comité de la Amistad Mundial Entre los Niños) fundado en 1926, apoyada por la Commission on International Justice and Goodwill (Comisión de Justicia Internacional y Voluntad Libre) del Federal Council of the Churches of Christ in America (Concilio Federal de Iglesias Cristianas en América).

El primer proyecto (1927) que se llevó a cabo por parte de esta agrupación fue el envío de muñecas americanas, ${ }^{32}$ conocidas como blue-eyed dolls, o muñecas de ojos azules, a Japón durante el festival del Hinamatsuri, con el fin de trasladar un mensaje de buena voluntad, entendimiento y paz entre ambas naciones. ${ }^{33}$ En total, se reunieron doce mil setecientas treinta y nueve muñecas, cada una de ellas con su correspondiente pasaporte que decía: "Esta muñeca es una buena ciudadana de los Estados Unidos de América. Obedecerá todas las leyes y costumbres de tu país. Por favor, cuida de ella mientras esté contigo.", ${ }^{34}$ un billete de tren y barco, y en algunos casos, cartas escritas a mano tanto por parte de Gulick como de los niños que las enviaban. Finalmente, las muñecas partieron hacia el Monbusho (Departamento Japonés de Educación) en cinco barcos de vapor llegando a tiempo para el Hinamatsuri. ${ }^{35}$

A partir de este momento, se empezó a preparar una respuesta a este envío por parte de Japón. ${ }^{36}$ Considerando la falta de tiempo, se optó por preparar un total de cincuenta y ocho muñecas Ichimatsu-ningyō, a las que se denominó como Torei-ningyō (Muñecas de gratitud). De este modo, tenemos un total de cuarenta y siete muñecas en representación de las distintas prefecturas niponas, seis con los nombres de las más grandes ciudades japonesas (Tokio, Osaka, Kioto, Nagoya, Yokohama y Kobe), cuatro en representación de los territorios fuera del archipiélago en poder de Japón

\footnotetext{
Takaki, Ronald (1988).

30 Gordon, Bill (2014): Friendship Dolls. Their “mission of friendship” continues [página WEB]. http://www. bill-gordon.net/dolls/exch1927/historical/index.htm [Consulta: 23/01/2019].

31 Hirobe, Izumi (2001): pp. 8-9.

32 Committee on world friendship among children (1929): pp. 1-29.

33 Gordon, Bill (2014): Friendship Dolls. Their “mission of friendship” continues [página WEB]. http://www. bill-gordon.net/dolls/exch1927/gulick/index.htm [Consulta: 23/01/2019].

34 Traducción de la autora. Idem.

35 Idem.

36 Idem.
} 
(Taiwán, Chosen (Corea), Karafuto (Sakhalin), Kanto-shu (península de Liaotung)). Por último, la muñeca más importante y más cara era Miss Dai Nippon o Miss Japón.

A la hora de elaborar estos ejemplares, se llamó a los mejores y más expertos artesanos: sus atuendos se realizaron con las sedas más caras, adornados con patrones y estampados pintados a mano; cada uno de los ejemplares se acompañó de un pasaporte, cartas de los niños japoneses, un juego de té y otros accesorios, además de un libro ilustrado acerca de los niños y las fiestas del té en Japón. A su llegada a Estados Unidos, las muñecas se dividieron en un total de seis grupos, de modo que cada uno de ellos iba destinado a una región del país, igualmente, estos grupos se dividieron para dar cabida a las regiones más pequeñas. Las muñecas más bellas y delicadas fueron expuestas en museos y bibliotecas públicas por todo el país; sabemos con seguridad la localización de algunas de estas muñecas, como es el caso de Miss Japón, en el Smithsonian Museum, en Washington. ${ }^{37}$

Tristemente, 14 años después de este episodio estalló la II Guerra Mundial (19391945), momento en el que varias de estas muñecas se destruyeron, vendieron o perdieron por ser consideradas como una personificación del enemigo. Desde este momento hasta aproximadamente la década de los 80 del siglo pasado, no se ha sabido nada del destino de estas ningyō, sin embargo, a día de hoy, la mayoría de los ejemplares han sido localizados, salvando un total de catorce muñecas que todavía siguen perdidas. ${ }^{38}$

Centrándonos, ahora sí, en el caso español, vamos a encontrarnos, en primer lugar, toda una serie de notas referentes a exposiciones celebradas en España y dedicadas exclusivamente a este tipo de objetos. Un ejemplo que nos parece de especial interés nos traslada a una exposición que se llevó a cabo en Madrid en 1965, concretamente en El Corte Inglés, gracias a la dirección de Japan Air Lines en colaboración con Air France, a modo de celebración ante la aparición de vuelos Europa-Japón. ${ }^{39}$ Las piezas expuestas ya se habían mostrado con anterioridad en París, Génova, Milán y Zúrich, siendo un total de cuarenta y seis figuras de las más diversas tipologías. Según nos cuenta el artículo, estas muñecas representan personajes propios del teatro y el folclore japoneses dispuestas en tres escenarios cuya decoración emulaba un jardín japonés. Principalmente se trata de representaciones de geisha y bailarinas.

Si bien, no es el único caso, ya que podemos mencionar muestras posteriores, como las organizadas por la Japan Foundation, ${ }^{40}$ algunas de ellas centradas en las muñecas propiamente dichas mientras que otras las incorporan como un elemento más, dependiendo de la temática. A título de ejemplo, podemos mencionar aquellas tituladas El mundo de las Kokeshi o Muñecas del Japón, ${ }^{41}$ entre otras. Lo mismo se podría decir de aquellas que han tenido lugar en el Museu Etnològic de Barcelona, gran parte de ellas recogidas por la doctora Muriel Gómez Pradas en su tesis doctoral, y centradas en las colecciones de juguetes tradicionales que alberga dicho museo. No obstante, y como ella misma señala, a partir de la creación del Espai Japó (1998, actualmente extinto) podemos enumerar nuevas muestras de muy diversa temática donde las ningyō están presentes: es el caso de Itadakimasu. Cultura y ali-

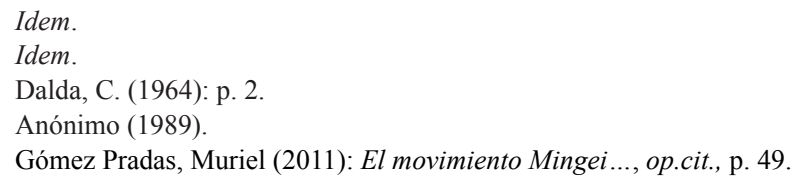


mentación en Japón o Japón y el mundo del manga, como algunos ejemplos, ${ }^{42}$ de los que también se hace eco la prensa. ${ }^{43}$ Otro ejemplo de institución que ha organizado exposiciones de ningyō y otras piezas es el Museu del Joguet, que cuenta con una amplia colección de juguetes de todo el mundo. Jugar al Japó (2008), Koï-Nobori. La festa dels nen japonesos (2011), ${ }^{44}$ Nines del món $(2016)^{45}$ son tres casos que este museo ha llevado a cabo.

A pesar de los ejemplos comentados, las exposiciones de muñecas niponas se extienden hasta llegar a la rabiosa actualidad, como pone de relieve la muestra que tuvo lugar en la Universidad de Salamanca con las creaciones de Hatsuko Ohno en 2017 o el caso de Madrid, que durante el mes de noviembre de 2018 acogió otro ejemplo, centrado, en este caso, en las piezas del Maestro Hisashige Ito XII, cuya tipología principal fueron las Gosho-ningyō o muñecas de palacio. ${ }^{46}$ Ambos ejemplos nos hablan de una puesta en valor y reconocimiento de algunos nombres propios dentro de la creación de ningyō, cada uno de los cuales se centra en tipologías distintas resaltando su valor y belleza.

Podemos citar también sucesos extraordinarios que atestiguan estos intercambios. Este es el caso de un episodio que tuvo lugar en Córdoba, en 1964, cuando una niña recibe una muñeca enviada desde Hiroshima como respuesta a una carta en la que solicitaba esta pieza al propio alcalde. ${ }^{47}$

Igualmente, no podemos dejar de señalar el hermanamiento entre la ciudad japonesa de Yamaguchi y la española Pamplona que se produjo en el año 1980. Esta relación queda atestiguada en el Museo de Pamplona gracias a uno de los regalos protocolarios que fueron intercambiados entre las dos ciudades: nada más y nada menos que una ningyō perteneciente a la tipología de Ishō-ningyō o muñecas de vestir que ya hemos definido al principio. Además, podemos encontrar otros testigos de esta unión, como puede ser el caso del pequeño jardín japonés que se encuentra en el parque Yamaguchi.

\section{Conclusiones}

En primer lugar y atendiendo a lo expuesto en los puntos anteriores, podemos señalar la consideración de estas muñecas tradicionales japonesas como manifestación artística, ya sea por su calidad y belleza o bien por ser testimonio de épocas pasadas con un cierto carácter exótico, por ello, además de despertar el interés de algunos coleccionistas, también fue protagonista de otro tipo de intercambios de carácter diplomático así como objeto de atención de otros medios como la prensa y la ilustración.

A lo largo del tiempo, se han establecido toda una serie de relaciones entre nuestro país y el archipiélago nipón, de distinta índole y con algunos momentos de mayor importancia. No obstante, nos interesa señalar que a lo largo de estos intercambios las muñecas japonesas han estado presentes en todo momento, y testigo de ello son

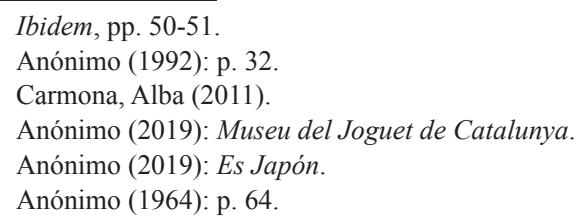


las piezas que a día de hoy se conservan en distintos museos e instituciones públicas y privadas de toda España. Por supuesto, debemos mencionar también el papel que tiene la prensa a la hora de documentar algunos de estos acontecimientos, siendo una fuente fundamental a la hora de profundizar en ellos además de ser una fuente para situarnos de primera mano en otros contextos interesantes para nuestra investigación.

Bien es verdad, como hemos reflejado en anteriores apartados, que quizá estas piezas han recibido una atención menor si las comparamos con otros objetos llegados a España al mismo tiempo, si bien, esperamos haber puesto de relieve su presencia que, aunque menor, ha formado parte de intercambios, noticias de prensa, reportajes, creaciones literarias y artísticas y, por supuesto, colecciones y exposiciones; siendo todo ello un vehículo para el conocimiento y difusión de las ningyō.

\section{Bibliografía}

Almazán Tomás, David (1999): Japón y el Japonismo en las revistas ilustradas españolas (1870 - 1935). Tesis doctoral dirigida por Elena Barlés Báguena, Departamento de Historia del Arte, Universidad de Zaragoza.

Almazán Tomás, David (2004): “El pintor José Blanco Coris (1862-1946) y su "Manual de Arte Decorativo" (1916): la enseñanza del arte extremo oriental y el fenómeno del "Japonismo" en España". En: Artigrama, 19 [número], Zaragoza, pp. 503-522.

Almazán Tomás, David (2011): “Arte japonés y japonismo en España”. En Cid, Fernando. (coord.): Japón y la Península Ibérica: cinco siglos de encuentros. Gijón: Satori, p. 251.

Anónimo (1888): "La muñeca japonesa", El Camarada. Semanario infantil ilustrado, Barcelona, 20/10/1888, pp. 813-814.

Anónimo (1906): “La vida contemporánea”, La Ilustración Artística, Barcelona, xx/12/1906, p. 778 .

Anónimo (1906): La Ilustración Artística, Barcelona, 8 /10/1906, p. 643.

Anónimo (1916): Nuevo Mundo, Madrid, 25 /08/1916.

Anónimo (1917): “Taller de Santa Rita”, Correo de la mañana, Badajoz, 17/08/1917.

Anónimo (1928): “Cosas de Japón. La boda del príncipe Chichibu”, La libertad, Madrid, 16/06/1928, p. 1.

Anónimo (1929): Nuevo Mundo, Madrid, 26/04/1929.

Anónimo (1929): La Esfera, Madrid, 27/04/1929.

Anónimo (1943): “Algo más sobre la historia de la muñeca”, Imperio: Diario de Zamora de Falange Española de las J.O.N.S., Zamora, 32/05/1943.

Anónimo (1964): "Muñeca japonesa para una niña de Córdoba". ABC, Madrid, 06/09/1964, p. 64.

Anónimo (1970): “Castellón es en estos días un polícromo bazar de juguetes”, Mediterráneo: Prensa y Radio del Movimiento, Castellón, 04/01/1970.

Anónimo (1989): “Espectáculos”. ABC, Madrid, 20/10/1989, p. 112. Anónimo (2018): Técnicas sublimes del arte japonés - Exposición itinerante de la Fundación Japón [página WEB]. https://www.fundacionjapon.es/es/Actividades/Arte-y-Cultura/evento/234/exposicion-tecnicas-sublimes-del-arte-japones [Consulta: 03/07/2019].

Anónimo (1992): “Cultura”. La Vanguardia, Barcelona, 16/11/1992, p. 32.

Anónimo (2019): Museu del Joguet de Catalunya. Exposició temporal [página WEB]: https://www.mjc.cat/agenda/nines-del-mon/ [Consulta: 03/07/2019]. 
Anónimo (2019): Es Japón [página WEB]. http://www.esjapon.com/exposicion-munecas-de-palacio-29683 [Consulta: 24/01/2019].

Barlés Báguena, Elena (2012): "El descubrimiento en Occidente de Japón y de sus artes durante la Era Meiji (1868-1912)". En Barlés Báguena, Elena, Almazán Tomás, David (eds.): La fascinación por el arte del País del Sol Naciente. El encuentro entre Japón y Occidente en la Era Meiji (1868-1912). Zaragoza: Fundación Torralba, Fundación Japón, Museo de Zaragoza, pp. 95-156.

Barlés Báguena, Elena (2013): "Presencia e impacto del arte japonés en España en la época del Japonismo (segunda mitad del siglo XIX y primeras décadas del XX). Un estado de la cuestión". En Boletín de Bellas Artes, XL, Sevilla, pp. 77-142.

Bru, Ricard (2007): “Els inicis del comerç d'art japonès a Barcelona (1868-1887)". En: Butlletí de la Reial Acadèmia catalana de Belles Arts de Sant Jordi, 21, Barcelona, p. 59.

Bru, Ricard (2013): “El Japón en la Exposición Universal de 1888”. En AA.VV., Japonismo. La fascinación por el arte japonés (cat.exp.). Madrid: CaixaForum, pp. 118-123.

Carmona, Alba (2011): "Nines i estels per recordar el sisme nipó”. Diari de Girona [página WEB]. https://www.diaridegirona.cat/cultura/2011/07/18/nines-estels-recordar-sisme-nipo/502065.html [Consulta: 03/07/2019].

Committee on world friendship among children (1929): Dolls of friendship; the story of a goodwill project between the children of America and Japan. Nueva York: Friendship Press.

Dalda, C. (1964): "La muñeca, reina de la juguetería española”. Hoja del Lunes, Granada, 06/01/1964, p. 2.

El Progreso: Diario liberal, Lugo, 24/12/1916.

Gómez Pradas, Muriel (2010): “La pervivencia en el Japón actual de festividades tradicionales: El caso del Hina Matsuri y el Tango-No-Sekku”. En Barlés Báguena, Elena, Almazán Tomás, David (coords.): Japón y el mundo actual. Zaragoza: Prensas universitarias de Zaragoza, pp. 417-433.

Gómez Pradas, Muriel (2011): El movimiento Mingei en las colecciones del Museu Etnològic de Barcelona. El caso de los kyōdo-gangu o juguetes populares y tradicionales japoneses. Tesis doctoral dirigida por Elena Barlés Báguena, Departamento de Historia del Arte, Universidad de Zaragoza, 2 vols.

Gordon, Bill (2014): Friendship Dolls. Their "mission of friendship" continues [página WEB]. http://www.bill-gordon.net/dolls/exch1927/historical/index.htm [Consulta: 23/01/2019].

Hardenberg (1904): "La educación japonesa y las fiestas escolares en Japón”, La ilustración artística : periódico semanal de literatura, Barcelona, 11/07/1904, p. 460.

Hirobe, Izumi (2001): Japanese Pride, American Prejudice: Modifying the Exclusion Clause of the 1924 Immigration Act. Standford: Stanford University Press, pp. 8-9.

Pate, Alan Scott (2005): Ningyō. The art of the japanese doll. Singapur: Tuttle Publishing

Pate, Alan Scott (2016): Art As Ambassador: The Japanese Friendship Dolls of 1927. Antique Japanese Dolls.

Pate, Alan Scott: "Sosaku Ningyō: The Birth of The Japanese Art Doll”. En Antique Japanese Dolls. http://www.antiquejapanesedolls.com/pub_artinfocus/sosaku_ningyo/sosaku_ningyo.html [Consulta: 23/01/2018].

The Japan Chronicle (1916): "Diversiones de las japonesas".

Shoaf, Judy (2003): The Japanese doll on the Western Toyshelf [página WEB], http://users. clas.ufl.edu/jshoaf/Jdolls/jdollwestern/2005/index.html [Consulta: 23/01/2019].

Takaki, Ronald (1988): Strangers from a Different Shore: A History of Asian Americans. 
Boston: Little, Brown.

Whitney, John (ed.) (1991): The Cambridge History of Japan. Nueva York: Cambridge University Press, vol. 4.

Yamada, Tokubei (1955): Japanese Dolls. Tokio: Japan Travel Bureau. 